\title{
Over-the-counter Medication Sales Surveillance for Early Detection of Respiratory Epidemics in Rural China
}

\author{
Qin Qin', Liwei Cheng1, Li Tan¹, Yunzhou Fan¹, Li Liu', Lihong Tian'1, Ying Wang¹, Hongbo \\ Jiang ${ }^{1}$, Sheng Wei ${ }^{1}$, Vinod K. Diwan², Weirong Yan ${ }^{1,2}$ and Shaofa Nie*1
}

${ }^{1} T o n g j i$ Medical College, Huazhong University of Science and Technology, Wuhan, China; ${ }^{2}$ Division of Global Health (IHCAR), Department of Public Health Science,Karolinska Institutet, Stockholm, Sweden

\section{Objective}

To explore the feasibility of using OTC medication sales data for early detection of respiratory epidemics in rural China

\section{Introduction}

Respiratory infectious diseases are the most common diseases reported in rural China. Studies have suggested that the OTC retail sale data could be used to detect early outbreak $(1,2)$. However, few researches have performed to identify whether OTC retail sales data could also predict the outbreak in developing countries and resource poor settings. Here, we conducted a web-based syndromic surveillance system with OTC retail sales to detect respiratory epidemics in rural area in China.

\section{Methods}

80 kinds of drugs for respiratory infectious diseases have been selected including 12 antibiotics, 5 antipyretics, 30 compound cold medicine, and 33 cough suppressants. From Apr. 1st to Sep. 30th 2012, sales data were collected from 23 pharmacies in two rural counties in Hubei Province, central China, by the web-based surveillance system. Data of all respiratory infectious cases were obtained by the China Information System for Diseases Control and Prevention (CISDCP) in same region and during the same period. The-day-of-the week effect was adjusted and then time series of adjusted data were compared to explore the correlation.

\section{Results}

Compound cold medicines combined with different ingredients including antipyretics, vitamins and Chinese herbal medicine accounted for nearly $50 \%$ of total sales volume, while cough suppressants and antibiotics accounted for nearly $25 \%$ separately. The adjusted data were capable of fitting the original data. Time trend of different categories were similar to each other. However, no correlation was found between sales data and number of respiratory infectious cases (Figure $1)$.

\section{Conclusions}

The results did not support the surveillance of OTC medicines sales could detect epidemics of respiratory infectious disease. However, it was tentative due to multiple factors like no sizable outbreaks during the period and short term data for analysis might influence this outcome. More studies are needed to further investigate the feasibility of using OTC medication sales data for the early outbreak detection.

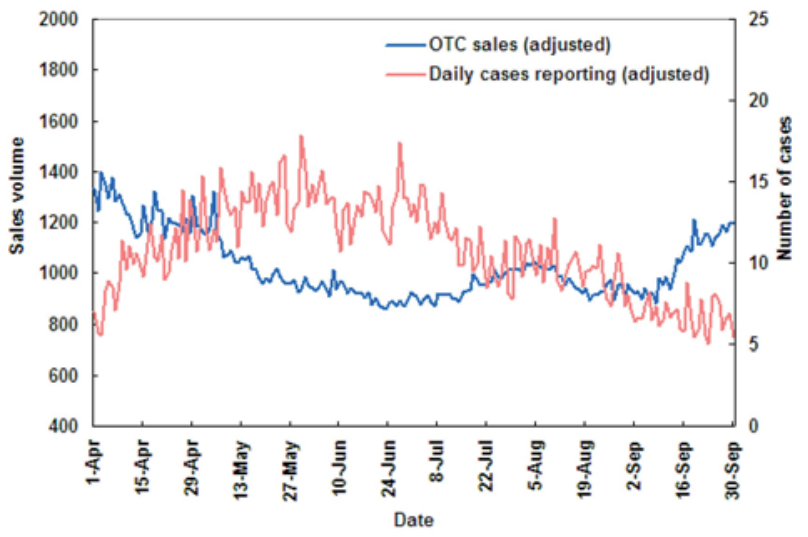

Figure 1 Comparison of ОTC medicines sales (adjusted) with number of respiratory infectious cases reported in the CISDCP system, by date,

April to September, 2012

\section{Keywords}

Medication sales; Respiratory epidemics; Surveillance

\section{Acknowledgments}

The study is financially supported by a grant under the European Union Framework Program 7 (project no: 241900).

\section{References}

1. Magruder S. Evaluation of over-the-counter pharmaceutical sales as a possible early warning indicator of human disease. Johns Hopkins APL technical digest 2003; 24(4):349-53.

2. Das D, Metzger K, Heffernan R, Balter S, Weiss D, Mostashari F. Monitoring over-the-counter medication sales for early detection of disease outbreaks-New York City. MMWR 2005; 54 Suppl: 41-6.

\section{*Shaofa Nie}

E-mail: sf_nie@mails.tjmu.edu.cn 